\title{
On the Effects of the Interaction Potential Parameters on Bose-Einstein Condensation
}

\author{
M.K. Al-Sugheir*, H.A. Al-Khzon, M. Al-Maghrabi And G.A. Alna’washi \\ Department of Physics, the Hashemite University, Zarqa, Jordan
}

(Received March 26, 2012)

\begin{abstract}
In this work the effect of the potential parameters on the condensate fraction and the critical temperature of neutral many-bosonic system are investigated. A many-body technique called the static fluctuation approximation is used in this study. The interaction potential is modeled by two linear terms. The condensation fraction and critical temperature were found to decrease with increasing the strength or the range of the repulsive part of the potential. On the other hand, the condensation fraction and critical temperature increase with increasing the depth or range of the attractive part of the potential.
\end{abstract}

PACS: 67.10.Ba, 67.25.bd, 51.30.+i

\section{Introduction}

The Bose-Einstein condensation (BEC) is one of the fascinating quantities for neutral many-bosonic systems. Determination of the critical temperature and condensate fraction at finite temperature of such systems is one of the challenges for the many-body approaches. Most of the many-body approaches are well established at low temperatures or for weakly interacting systems. In this work we shall use the static fluctuation approximation (SFA) approach [1] to calculate the condensate fraction and critical temperature for neutral many-bosonic system. The role of the potential parameters, the potential strength of the repulsive part, range of the repulsive interaction, depth of the attractive part, and range of the attractive potential on these quantities are investigated.

The neutral many-bosonic systems are characterized by extraordinary properties. Many theoretical techniques and formalisms were applied to explain their observed behavior. Nevertheless, some basic properties of these systems remain not well understood. Such systems reveal quantum-mechanical effects on a macroscopic scale (e.g. superfluidity and BEC) and hydrodynamics on a microscopic scale (vortices; quantum hydrodynamics). The relation, if any, between BEC and superfluidity remains unclear.

In the present work we shall consider an extended system of $N$ particles each of mass $m$, occupying a volume $\Omega$. Further, we shall assume that these particles interact with each other through a model potential consisting of two linear parts. Such a model potential gives us an opportunity to study the effect of the various potential parameters on condensate fraction and critical temperature of the system.

The SFA adopted in this study has already been applied to weakly and strongly many-body systems [1-8]. This approach is relatively simple compared to other many-body approaches in the sense that it is not based

\footnotetext{
* corresponding author; e-mail: msugh@hu.edu.jo
}

on Green's functions or Feynman's diagrams. Despite the simplicity of the approach, it is applicable, at least in principle, to any arbitrary system. It is based on the replacement of the square of the local field operator by its mean value. The physical implication is that the true quantum-mechanical spectrum of the local-field operator is replaced by a distribution around its expectation value. In fact, this is the only approximation that appears in the SFA.

In a previous work, a simple step potential model is used to investigate the role of the repulsive interaction on such systems [7]. This work is a continuation for that with more realistic potential consisting of repulsive and attractive potential. The strength of the potential naturally affects the condensate fraction, critical temperature and other properties of the system. This topic was studied by many workers $[9,10]$.

For the ideal Bose gas, the condensate fraction is $100 \%$ at absolute zero temperature. Of course, the amount of condensation will be different when the potential is switched on. The condensate fraction for weakly interacting systems is greater than that for strongly interacting systems [11] such as liquid ${ }^{4} \mathrm{He}$, where the condensate fraction was down to $10 \%$. Also, the potential effect on the critical temperature can immediately be seen from the fact that the critical temperature in liquid ${ }^{4} \mathrm{He}$ is $\approx 2.17 \mathrm{~K}$; whereas the corresponding theoretical value calculated for an ideal Bose gas whose constituent bosons have a ${ }^{4} \mathrm{He}$-mass is $3.14 \mathrm{~K}[12,13]$.

There is a growing interest in BEC and thermodynamic properties of bosonic systems $[14,15]$. The BEC plays an important role in the theory of physical systems; it is masked either by a strong interaction or by the complexities of the system [16].

Various microscopic techniques have been used over the years to study neutral many bosonic systems such as the single-particle Green-function method [17], the variational track which includes the highly acclaimed correlated-basis-function method [18] and the density-functional approach [19]. 
Due to the cooling development of experimental techniques, the first gases to be cooled to degeneracy temperatures and display BEC were alkali gases such as rubidium [20], sodium [21] and lithium [22]. Later, BEC was obtained in spin-polarized hydrogen atoms [23]; metastable ${ }^{4} \mathrm{He}[24]$ and other atomic species are also under active investigation for Bose condensation.

Following the introduction, the SFA formalism is briefly presented in Sect. 2 for a neutral many-bosonic system. Section 3 is devoted for results and discussion. Summary and concluding remarks are presented in Sect. 4.

\section{Static fluctuation approximation}

The basic assumptions in SFA are based on [1]: the square of the local-field operator fluctuation $\Delta \hat{E}_{\boldsymbol{k}}^{2}$ can be replaced by its mean value $\Delta \hat{E}_{\boldsymbol{k}}^{2}=\left\langle\Delta \hat{E}_{\boldsymbol{k}}^{2}\right\rangle=\Phi_{\boldsymbol{k}}^{2}$, the Hamiltonian of the system can be written as a linear combination of a Hermitian local-field operator $\hat{E}_{\boldsymbol{k}}$ and the occupation number operator $\hat{n}_{\boldsymbol{k}}$ :

$$
\hat{H}=\sum_{\boldsymbol{k}} \hat{E}_{\boldsymbol{k}} \hat{n}_{\boldsymbol{k}}
$$

where the summation is over all states, the local-field operator commutes with the creation and annihilation operators.

In this section we will present briefly the basic elements of the SFA for neutral many bosonic systems. In the Heisenberg picture, the time evolution of the creation operator $\hat{b}_{\boldsymbol{k}}^{+}(\tau)$ is given by

$$
\hat{b}_{k}^{+}(\tau)=\exp (\tau \hat{H}) \hat{b}_{k}^{+}(0) \exp (-\tau \hat{H}),
$$

where $\tau \equiv \mathrm{i} t, \boldsymbol{k}$ is the wave number describing a specific state and $\hat{H}$ is the Hamiltonian. The equation of motion for the creation operator in this picture can be written as

$$
\frac{\mathrm{d} \hat{b}_{\boldsymbol{k}}^{+}(\tau)}{\mathrm{d} \tau}=\left[\hat{H}, \hat{b}_{\boldsymbol{k}}^{+}(\tau)\right] .
$$

Based on the SFA assumptions, the equation of motion becomes

$$
\frac{\mathrm{d} b_{\boldsymbol{k}}^{+}(\tau)}{\mathrm{d} \tau}=\left[\hat{H}, b_{\boldsymbol{k}}^{+}\right]=\hat{E}_{\boldsymbol{k}} b_{\boldsymbol{k}}^{+} .
$$

The local-field operator $\hat{E}_{\boldsymbol{k}}$ calculated from Eq. (4) is

$$
\hat{E}_{\boldsymbol{k}}=\left[\hat{b}_{\boldsymbol{k}},\left[\hat{H}, \hat{b}_{\boldsymbol{k}}^{+}\right]\right] \text {. }
$$

The physical quantities: occupation number, mean value of the local-field operator, and fluctuation in the local-field operator, can be calculated by the aid of the SFA. To evaluate these quantities based on the SFA, the grand Hamiltonian in second quantization of the system should be determined. The grand Hamiltonian describing the neutral many-bosonic systems in the second quantization can be written as [1]:

$$
\hat{H}=\sum_{\boldsymbol{k}}\left(\frac{\hbar^{2} k^{2}}{2 m}-\mu\right) \hat{b}_{\boldsymbol{k}}^{+} \hat{b}_{\boldsymbol{k}}+\frac{1}{2} \sum_{\boldsymbol{k}} V(k) \hat{\rho}_{\boldsymbol{k}} \hat{\rho}_{-\boldsymbol{k}},
$$

where $V(k)=\int V(r) \exp (\mathrm{i} \boldsymbol{k} \cdot \boldsymbol{r}) \mathrm{d} \boldsymbol{r}, \hat{\rho}_{\boldsymbol{k}} \equiv \frac{1}{\Omega} \sum_{\boldsymbol{q}} \hat{b}_{\boldsymbol{k}+\boldsymbol{q}}^{+} \hat{b}_{\boldsymbol{q}}$, and $\mu$ is the chemical potential of the system.
The local-field operator, $\hat{E}_{k}$, is calculated from Eqs. (5) and (6) to be

$$
\hat{E}_{\boldsymbol{k}}=\left[\hat{b}_{\boldsymbol{k}},\left[\hat{H}, \hat{b}_{\boldsymbol{k}}^{+}\right]\right]=\varepsilon(k)+\frac{1}{\Omega} \sum_{\boldsymbol{q}} W(\boldsymbol{k}, \boldsymbol{q}) \hat{n}_{q},
$$

where $W(\boldsymbol{k}, \boldsymbol{q}) \equiv V(0)+V(\boldsymbol{k}-\boldsymbol{q})$ and $\varepsilon(k)=\frac{\hbar^{2} k^{2}}{2 m}-\mu$. The other quantities can be calculated from the generalized long-range equation of the system given by [1]:

$$
\left\langle\hat{n}_{\boldsymbol{k}} \hat{A}\right\rangle=\eta_{0}(\boldsymbol{k})\langle\hat{A}\rangle+\eta_{1}(\boldsymbol{k})\left\langle\Delta \hat{E}_{\boldsymbol{k}} \hat{A}\right\rangle,
$$

where

$$
\begin{gathered}
\eta_{0}(k) \equiv \frac{1}{2}\left[\frac{1}{\exp \left(\beta\left(\left\langle\hat{E}_{k}\right\rangle+\varphi_{k}\right)\right)-1}\right. \\
\left.+\frac{1}{\exp \left(\beta\left(\left\langle\hat{E}_{k}\right\rangle-\varphi_{k}\right)\right)-1}\right], \\
\eta_{1}(k) \equiv \frac{1}{2 \varphi_{k}}\left[\frac{1}{\exp \left(\beta\left(\left\langle\hat{E}_{k}\right\rangle+\varphi_{k}\right)\right)-1}\right. \\
\left.-\frac{1}{\exp \left(\beta\left(\left\langle\hat{E}_{k}\right\rangle-\varphi_{k}\right)\right)-1}\right]
\end{gathered}
$$

and $\hat{A}$ is an arbitrary operator commuting with local-field operator, creation, and annihilation operators.

We can get the occupation number of particles by substituting $\hat{A}=1$ in Eq. (8) yielding

$$
\left\langle\hat{n}_{\boldsymbol{k}}\right\rangle=\eta_{0}(\boldsymbol{k})+\eta_{1}(\boldsymbol{k})\left\langle\Delta \hat{E}_{\boldsymbol{k}}\right\rangle \text {. }
$$

The mean value of the fluctuation is zero; hence the above equation takes the following form:

$$
\left\langle\hat{n}_{\boldsymbol{k}}\right\rangle=\eta_{0}(\boldsymbol{k}) \text {. }
$$

It is more convenient to rewrite the long-range equation in terms of the deviation of the occupation number operator, defined as:

$$
\Delta \hat{n}_{\boldsymbol{k}} \equiv \hat{n}_{\boldsymbol{k}}-\left\langle\hat{n}_{\boldsymbol{k}}\right\rangle \text {. }
$$

So the long-range equation can be written as

$$
\left\langle\Delta \hat{n}_{\boldsymbol{k}} \hat{A}\right\rangle=\eta_{1}(\boldsymbol{k})\left\langle\Delta \hat{E}_{\boldsymbol{k}} \hat{A}\right\rangle \text {. }
$$

The equation describing the pair correlation function can be obtained by substituting $\hat{A}=\Delta \hat{n}_{\boldsymbol{q}}$ in Eq. (14) with $\boldsymbol{k} \neq \boldsymbol{q}$ :

$$
\begin{aligned}
& \left\langle\Delta \hat{n}_{\boldsymbol{k}} \Delta \hat{n}_{\boldsymbol{q}}\right\rangle_{\mathrm{c}}=\eta_{1}(\boldsymbol{k})\left\langle\Delta \hat{E}_{\boldsymbol{k}} \Delta \hat{n}_{\boldsymbol{q}}\right\rangle, \\
& \left\langle\Delta \hat{n}_{\boldsymbol{k}} \Delta \hat{n}_{\boldsymbol{q}}\right\rangle_{\mathrm{c}}=\frac{\eta_{1}(\boldsymbol{k})}{\Omega} \sum_{\boldsymbol{p}} W(\boldsymbol{k}, \boldsymbol{p})\left\langle\Delta \hat{n}_{\boldsymbol{p}} \Delta \hat{n}_{\boldsymbol{q}}\right\rangle .
\end{aligned}
$$

The square of the fluctuation in the number of particles cannot be calculated from the long-range equation, because $\hat{A}=\Delta \hat{n}_{k}$ does not commute with the creation or annihilation operators. The square of the fluctuation in the occupation number of particles is given as [1]:

$$
\begin{aligned}
& \left\langle\left(\Delta \hat{n}_{\boldsymbol{k}}\right)^{2}\right\rangle=\left\langle\hat{n}_{\boldsymbol{k}}\right\rangle\left(1+\left\langle\hat{n}_{\boldsymbol{k}}\right\rangle\right) \\
& +\frac{2 \eta_{1}(\boldsymbol{k})}{\Omega} \sum_{\boldsymbol{p}} W(\boldsymbol{k}, \boldsymbol{p})\left\langle\Delta \hat{n}_{\boldsymbol{p}} \Delta \hat{n}_{\boldsymbol{k}}\right\rangle .
\end{aligned}
$$


By substituting $\hat{A}=\Delta \hat{E}_{\boldsymbol{k}}$ in Eq. (14) we can obtain the fluctuations in the local-field operator

$$
\eta_{1}(\boldsymbol{k}) \varphi_{\boldsymbol{k}}^{2}=\frac{1}{\Omega} \sum_{\boldsymbol{p}} W(\boldsymbol{k}, \boldsymbol{p})\left\langle\Delta \hat{n}_{\boldsymbol{k}} \Delta \hat{n}_{\boldsymbol{p}}\right\rangle .
$$

To complete the closed set of nonlinear integral equations the chemical potential must be calculated from the condition

$$
N=\sum_{k}\left\langle n_{\boldsymbol{k}}\right\rangle
$$

The energy levels for infinite system are close to each other, so that the summation in the nonlinear integral Eqs. (7), (15)-(18) can be replaced by integration: $\sum_{k} \longrightarrow \frac{\Omega}{(2 \pi)^{3}} \int \mathrm{d} \boldsymbol{k}$. The closed set of nonlinear integral Eqs. (7), (12), (15)-(18), can be solved numerically by an iterative method. Throughout this work a natural system of units will be used. In this system of units we set $\hbar=1=m$. The ${ }^{4} \mathrm{He}$ Bose system will be considered through the numerical calculation, so the conversion factor will be $\frac{\hbar^{2}}{m}=12.120048 \mathrm{~K} \AA^{2}$ where $m$ is the ${ }^{4} \mathrm{He}$ atomic mass.

The interaction potential model used in this work is given by

$$
V(r)= \begin{cases}a_{1} r+a_{2}, & r<r_{1}, \\ b_{1} r+b_{2}, & r_{1}<r<r_{2} .\end{cases}
$$

The model potential consists of two linear terms. The first term starts from $r \rightarrow 0$ to the equilibrium position at $r_{1}$ with maximum potential strength $a_{2}$ at $r=0$. The other term starts from $r_{1}$ to vanishing potential at $r_{2}$. The potential depth was found to be $d=a_{1} r_{1}+a_{2}=$ $b_{1} r_{1}+b_{2}$. According to the continuity condition of the potential and $V(r)$ vanishing at $r=r_{2}$, we have the potential parameters relations

$$
b_{1}=\frac{d}{r_{1}-r_{2}}, a_{1}=\frac{d-a_{2}}{r_{1}} .
$$

In this work, the effect of the repulsive potential strength $a_{2}$, potential depth $d$, equilibrium position $r_{1}$, and attractive potential range $r_{2}$ on the condensate fraction and on the critical temperature is studied.

\section{Results and discussion}

The effect of the strength of the repulsive part of the potential on the condensate fraction and critical temperature is studied. $r_{1}$ and $r_{2}$ are taken as constants of values of $3.0 \AA$ and $5.0 \AA$, respectively and the potential depth is taken to be $d=-0.01 a_{2}$. The condensate fraction as a function of temperature for different values of $a_{2}$ is shown in Fig. 1. These results indicate that the strength of the repulsive part does not affect the condensate fraction in the ground state. At low temperatures, the system under this potential model behaves like an ideal Bose gas. This means that at low temperatures, the contribution of the interaction term on the energy spectrum of the system is constant and just shifts the chemical potential of the system. As the temperature increases the effect of the potential strength arises, where the condensate fraction decreases with increasing the potential strength. The transition from the condensate state to the excited state occurs smoothly in the ideal Bose gas $\left(a_{2}=0 \mathrm{~K}\right)$. As the potential strength increases this transition occurs abruptly at large potential strengths $\left(a_{2}=25 \mathrm{~K}\right)$. This effect of the potential strength is expected because as the repulsive part strength increases the inter-particle spacing increases. In such situation the de Broglie wavelength becomes smaller than the inter-particle spacing, which reduces the probability of overlapping between the waves associated with adjacent particles. As a result, the condensate fraction decreases.

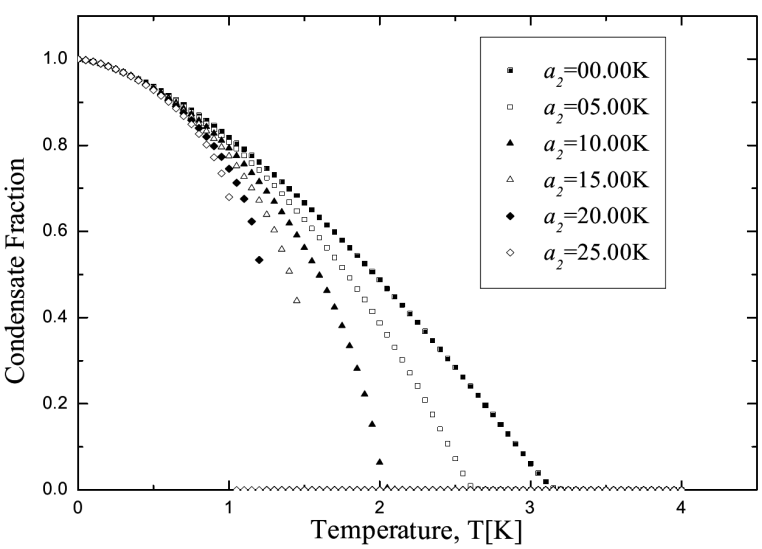

Fig. 1. The condensate fraction as a function of temperature calculated at $r_{1}=3.0 \AA, r_{2}=5.0 \AA, d=$ $-0.01 a_{2}$ for different values of $a_{2}$.

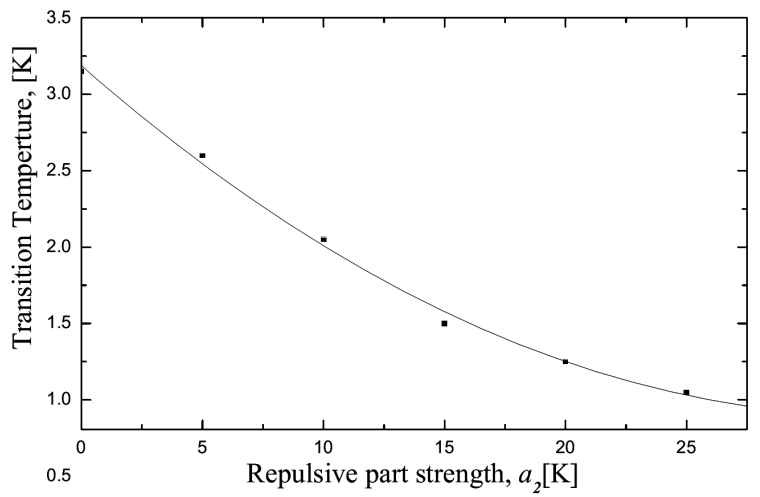

Fig. 2. The critical temperature as a function of the repulsive part strength $a_{2}$, at $r_{1}=3.0 \AA ; r_{2}=5.0 \AA$; and $d=-0.01 a_{2}$.

The critical temperature (the temperature at which the condensate fraction vanishes) at different values of potential strength $a_{2}$ is determined from Fig. 1 and displayed in Fig. 2 as a function of $a_{2}$. The results show that the critical temperature, $T_{\mathrm{c}}$, decreases as the strength of the repulsive part of the potential increases. Also, the variation in the critical temperature becomes smaller for higher repulsive part strengths. This means that the re- 
pulsive part of the potential lowers the critical temperature and transforms the system to the excited state at lower temperatures.

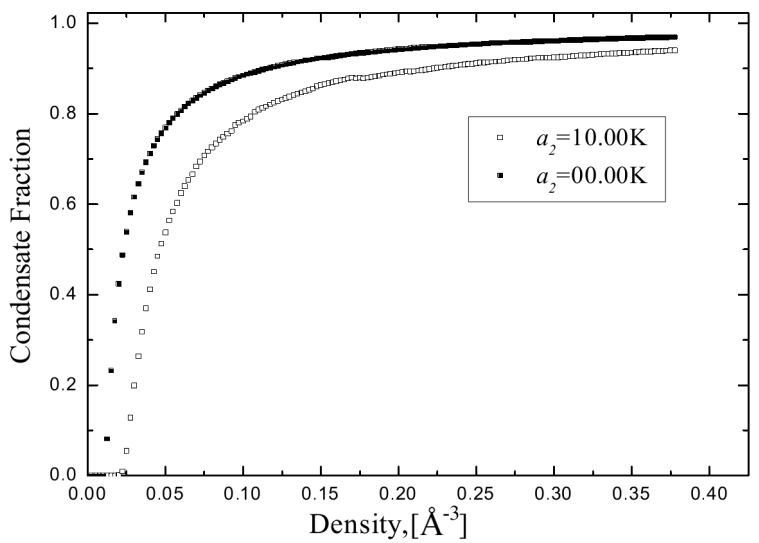

Fig. 3. The condensate fraction as a function of density at $r_{1}=3 \AA, r_{2}=5.0 \AA, d=-0.01 a_{2}$, and $T=2.05 \mathrm{~K}$ for two values of $a_{2}$.

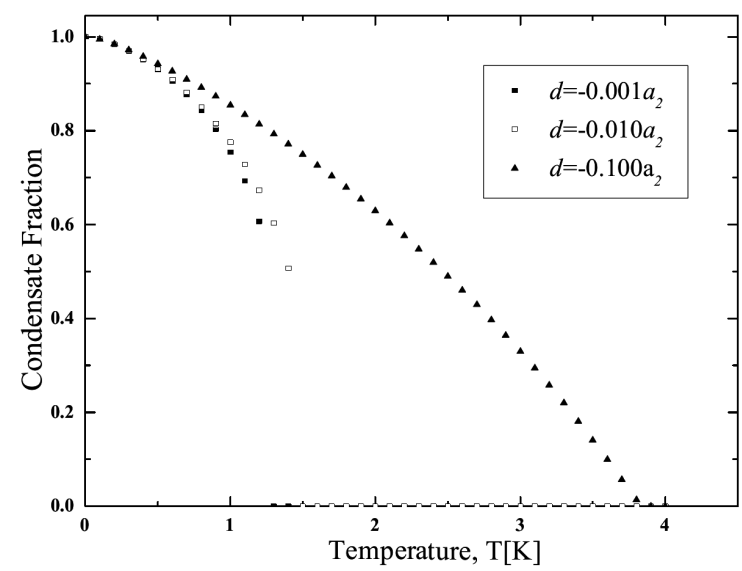

Fig. 4. The condensate fraction as a function of temperature $T$ at $a_{2}=15.0 \mathrm{~K}, r_{1}=3.0 \AA$, and $r_{2}=5.0 \AA$, for different depths of the attractive part.

Figure 3 shows the density dependence of the condensate fraction of the system. It is noted that the interacting system based on the used potential model behaves like an ideal gas. At low density, the condensate fraction increases rapidly reaching a saturation value at a certain density called the saturation density. This result is expected because BEC occurs if the de Broglie wavelength is comparable or greater than the inter-particle spacing. As the density of the system increases the inter-particle spacing decreases and the probability of overlapping between de Broglie waves associated with each particle increases.

The role of the potential depth on the condensate fraction was examined and the results are shown in Fig. 4. As expected, at low temperature the interaction potential does not play any role on the condensate fraction.
In other words the system behaves like an ideal Bose gas system. Contrary to the repulsive part of the potential strength, the condensate fraction and the critical temperature increase as the potential depth increases. In addition, the transition from the condensation state to the excited state occurs smoothly as the potential depth increases. The attractive interaction between the particles increases as the potential depth increases. This interaction reduces the inter-particle spacing, and hence increases the critical temperature and condensate fraction.

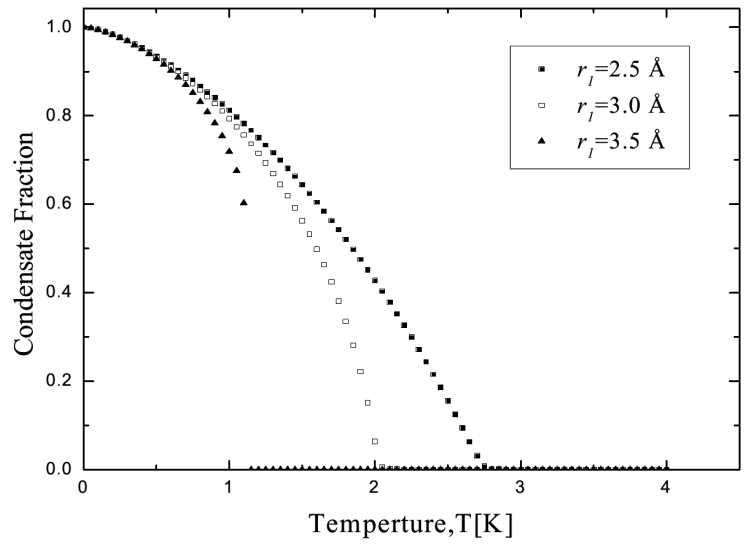

Fig. 5. The condensate fraction as a function of temperature $T$ at $a_{2}=10.0 \mathrm{~K}, r_{2}=5.0 \AA, d=-0.01 a_{2}$, for different values of $r_{1}$.

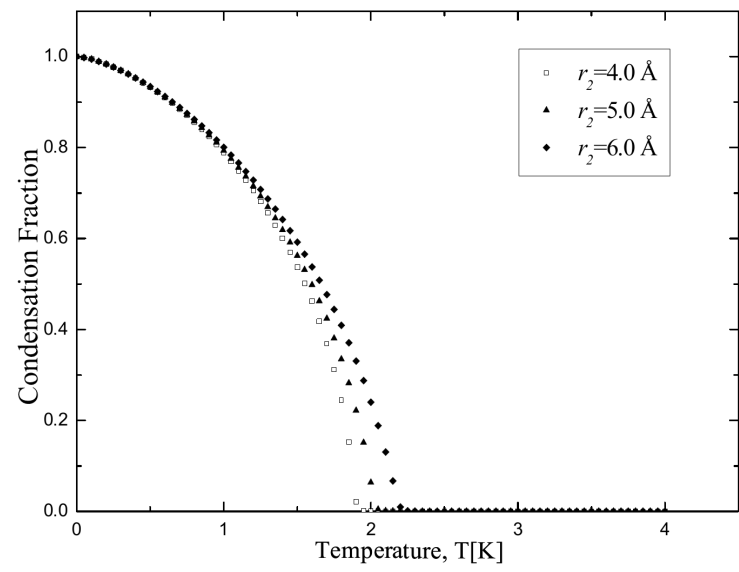

Fig. 6. The condensate fraction as a function of temperature $T$ at $a_{2}=10.0 \mathrm{~K}, r_{1}=3.0 \AA, d=-0.01 a_{2}$, for different values of $r_{2}$.

The effect of the equilibrium position on the condensate fraction was examined. Figure 5 shows the results for the condensate fraction as a function of temperature. As $r_{1}$ increases, both the critical temperature and the condensate fraction decrease. This result is in accordance with that for the potential strength of the repulsive part. In general we found that not only the strength of the repulsive part affect the condensate fraction, but also, the 
range of this part plays a major role on the condensate fraction and the critical temperature.

To explore the role of each term in the potential, the effect of the range of the attractive part $r_{2}$ is also studied. From Fig. 6 we observed that as the range of the attractive part of the potential increases the critical temperature and the condensate fraction increase. The increase in the attractive part range means that the particles of the system become closer to each other, and the inter-particle spacing becomes small. This would increase the probability of overlapping between the thermal wavelengths of each particle.

\section{Conclusions}

In this work, a model potential was used to describe a neutral many-bosonic system. The static fluctuation approximation was applied to calculate the condensate fraction and critical temperature. The role of the strength and range of the repulsive part of the potential as well as the effect of the depth and range of the attractive part of the potential on these quantities were investigated.

We may summarize the above results by saying that the increase in the strength or range of the repulsive part of the potential decreases the critical temperature and condensate fraction. In contrast, the increase in the depth or range of the attractive part increases the critical temperature and the condensate fraction.

\section{References}

[1] M.K. Al-Sugheir, H.B. Ghassib, R.R. Nigmatullin, Int. J. Theor. Phys. 40, 1033 (2001).

[2] M.K. Al-Sugheir, H.B. Ghassib, Int. J. Theor. Phys. 41, 705 (2002).

[3] M.K. Al-Sugheir, Int. J. Theor. Phys. 43, 1527 (2004).

[4] N.M. Ghulam, H.B. Ghassib, M.K. Al-Sugheir, Phys. Rev. C 75, 064317 (2007).

[5] B.R. Joudeh, M.K. Al-Sugheir, H.B. Ghassib, Int. J. Mod. Phys. B 19, 3987 (2005).
[6] A.S. Sandouqa, M.K. Al-Sugheir, H.B. Ghassib, Int. J. Theor. Phys. 45, 159 (2006).

[7] M.K. Al-Sugheir, S.S. Gasymeh, M. Shatnawi, M.S. Bawa'aneh Acta Phys. Pol. A 116, 154 (2009).

[8] M.K. Al-Sugheir, H.B. Ghassib, M. Awawdeh, Phys. Rev. A 84, 013617 (2011).

[9] K.A. Brueckner, K. Sawada, Phys. Rev. 102, 1117 (1957).

[10] K.A. Brueckner, K. Sawada, Phys. Rev. 106, 1128 (1957).

[11] N. Bogoliubov, in: The Many-Body Problem, Ed. D. Pines, Benjamin, London 1962, p. 292.

[12] K. Huang, Statistical Mechanics, 2nd ed. Wiley, New York 1987

[13] R.K. Pathria, Statistical Mechanics, 3rd ed., Pergamon Press, Oxford 1992.

[14] F. Dalfovo, S. Giorgini, L.P. Pitaevskii, S. Stringari, Rev. Mod. Phys. 71, 463 (1999).

[15] M. Kobayashi, M. Tsubota, Phys. Rev. B 66, 174516 (2002).

[16] L.P. Pitaevskii, Phys. Usp. 41, 569 (1998).

[17] S.S. Girish, C. Yia-Chung, Phys. Rev. B 57, 15144 (1998).

[18] E. Feenberg, Theory of Quantum Fluids, Academic Press, New York 1969.

[19] F. Dalfovo, A. Lastri, L. Pricaupenko, S. Stringari, G. Treiner, Phys. Rev. B 52, 1193 (1995).

[20] M.H. Anderson, J.R. Ensher, M.R. Matthews, C.E. Wieman, E.A. Cornell, Science 269, 198 (1995).

[21] K.B. Davis, M.O. Mews, M.R. Andrews, N.J. Van Druten, D.S. Durfee, N.M. Kurn, V. Ketterle, Phys. Rev. Lett. 75, 3969 (1995).

[22] C.C. Bradley, C.A. Sackett, J.J. Tollett, R.G. Hulet, Phys. Rev. Lett. 75, 1687 (1995).

[23] D.G. Fried, T.C. Killian, L. Willmann, D. Landhuis, S.C. Moss, D. Kleppner, T.J. Greytak, Phys. Rev. Lett. 81, 3811 (1998).

[24] F. Pereira Dos Santos, J. Leonard, J. Wang, C.J. Barrelet, F. Perales, E. Rasel, C.S. Unnikrishnan, M. Leduc, C. Cohen, Phys. Rev. Lett. 86, 3459 (2001). 